\title{
Mapping the Binding and Calmodulin-Dependent Activation of Nitric Oxide Synthase Isozymes
}

\author{
Donald E. Spratt, Yay Duangkham, Valentina Taiakina and J. Guy Guillemette*
}

Department of Chemistry, University of Waterloo, Waterloo, Ontario N2L 3G1, Canada

\begin{abstract}
Calmodulin $(\mathrm{CaM})$ is a ubiquitous cytosolic $\mathrm{Ca}^{2+}$-binding protein that can bind and activate numerous intracellular target proteins including the nitric oxide synthase (NOS) enzymes. There are three different isoforms of NOS found in mammals - neuronal NOS (nNOS), endothelial NOS (eNOS), and inducible NOS (iNOS) - with all three isozymes capable of producing the free radical, nitric oxide $(\bullet \mathrm{NO})$. At elevated cellular $\mathrm{Ca}^{2+}$ concentrations, CaM is able to bind and activate nNOS and eNOS. In contrast, the iNOS isozyme is transcriptionally regulated and binds to CaM in the absence of $\mathrm{Ca}^{2+}$. To further investigate the differences in the association of $\mathrm{CaM}$ to the $\mathrm{Ca}^{2+}$-dependent and $\mathrm{Ca}^{2+}$ independent NOS isoforms, a variety of $\mathrm{CaM}$ mutants were employed. These included CaM-troponin $\mathrm{C}$ chimeras, CaM EF hand pair proteins, $\mathrm{CaM}$ mutants incapable of binding to $\mathrm{Ca}^{2+}$, methionine oxidized $\mathrm{CaM}$ mutants, phosphomimetic $\mathrm{CaM}$ mutants and central linker $\mathrm{CaM}$ mutants. By characterizing these $\mathrm{CaM}$ proteins using a variety of biochemical techniques, we have mapped the binding and the CaM-dependent activation of the NOS enzymes to study their effect on the different electron transfer steps within the NOS enzymes. In addition, fluorescence and Förster resonance energy transfer (FRET) were used to monitor the orientation and conformation of CaM when bound to the NOS peptides and enzymes. Combining these cumulative results, a working model describing the CaM-dependent regulation of iNOS is proposed.
\end{abstract}

Keywords: Calmodulin, Nitric oxide synthase, posttranslational modification, electron transfer.

\section{INTRODUCTION}

Calmodulin $(\mathrm{CaM})$ is a ubiquitous $17 \mathrm{kDa} \mathrm{Ca}^{2+}$-binding cytosolic protein involved in the binding and regulation of numerous target proteins [1]. CaM consists of two globular lobes with each containing a $\mathrm{Ca}^{2+}$-binding $\mathrm{EF}$ hand pair joined by a central linker region. When intracellular $\mathrm{Ca}^{2+}$ concentrations increase, the binding of $\mathrm{Ca}^{2+}$ to $\mathrm{CaM}$ induces conformational changes in both lobes causing the increased exposure of hydrophobic pockets that enable $\mathrm{CaM}$ to associate with target proteins. The central linker of $\mathrm{CaM}$ also plays an important role in the binding and activation of its target proteins by acting as a flexible tether to allow for the correct orientation of the two globular lobes to associate to its targets [2]. CaM is also able to bind and regulate various intracellular target proteins at very low $\mathrm{Ca}^{2+}$ concentrations, making this association $\mathrm{Ca}^{2+}$-independent. The three mammalian nitric oxide synthase enzymes differ in their $\mathrm{Ca}^{2+}$-dependent and -independent regulation by CaM. This has allowed for more detailed investigations of these differences in their interactions with $\mathrm{CaM}$.

The nitric oxide synthase (NOS, EC 1.14.13.39) enzymes catalyze the production of nitric oxide $(\cdot \mathrm{NO})$, a reactive free radical that acts as a secondary inter- and intracellular messenger involved in many physiological processes [3].

\footnotetext{
*Address correspondence to this author at the Department of Chemistry, University of Waterloo, Waterloo, Ontario N2L 3G1 Canada;

Tel: 519-888-4567 ext. 35954; Fax: 519-746-0435;

E-mail: jguillem@uwaterloo.ca

Enzyme Commission Number: nitric oxide synthase (EC 1.14.13.39).
}

The $\cdot \mathrm{NO}$ is generated through two successive monooxygenation reactions requiring reduced nicotinamide adenine dinucleotidephosphate (NADPH) and molecular oxygen with L-citrulline as its biproduct [3]. Electrons originating from NADPH and molecular $\mathrm{O}_{2}$ are required for each of the mono-oxygenation steps. There are three NOS isozymes found in mammals - neuronal NOS (nNOS, NOS I), endothelial NOS (eNOS, NOS III) and inducible NOS (iNOS, NOS II). The NOS enzymes are homodimeric with each 135 to $160 \mathrm{kDa}$ monomer containing an $\mathrm{N}$-terminal oxygenase domain and a $\mathrm{C}$-terminal reductase domain. The oxygenase domain contains binding sites for the catalytic heme, tetrahydrobiopterin $\left(\mathrm{H}_{4} \mathrm{~B}\right)$, and the substrates Larginine and molecular oxygen, while the reductase domain binds the redox cofactors flavin mononucleotide (FMN), flavin adenine dinucleotide (FAD), and NADPH [4]. The oxygenase and reductase domains are separated by a CaMbinding domain. The binding of $\mathrm{CaM}$ to the NOS enzymes allows for efficient electron transfer from NADPH through the reductase domain to the heme for $\bullet \mathrm{NO}$ production [5]. The mechanism of how CaM binding facilitates electron transfer from NADPH to the heme is not fully understood and is not equivalent in all three NOS isoforms $[4,6,7]$. There is therefore a unique opportunity to investigate $\mathrm{Ca}^{2+} / \mathrm{CaM}$ control of these enzymes since $\cdot \mathrm{NO}$ production by each NOS isoform shows a different mode of activation and control by CaM. The nNOS and eNOS enzymes are constitutively expressed which is why they are collectively known as the constitutive NOS (cNOS) isoforms. The cNOS enzymes are $\mathrm{Ca}^{2+}$-dependent since their binding and activation are dependent on intracellular $\mathrm{Ca}^{2+}$ and $\mathrm{CaM}$ 
concentrations [4]. At elevated $\mathrm{Ca}^{2+}$ concentrations, CaM binds to the cNOS enzymes and promotes electrons originating from NADPH to be transferred through the reductase domain to the catalytic heme of the adjacent NOS monomer's oxygenase domain [5, 8-12]. There are three basic steps that make up the catalytic cycle of the reductase domain - (1) the hydride transfer from NADPH to FAD, (2) electron transfer from FAD to FMN, and (3) electron transfer from FMN to heme. Each of these electron transfer steps have been shown to be dependent on the conformation of the enzyme. In contrast to the cNOS enzymes, iNOS is transcriptionally regulated in vivo by cytokines in macrophages and binds to $\mathrm{CaM}$ at basal levels of $\mathrm{Ca}^{2+}[4]$. Since this enzyme binds to $\mathrm{CaM}$ regardless of cellular $\mathrm{Ca}^{2+}$ concentrations, this enzyme is classified as $\mathrm{Ca}^{2+}$ independent. Electron transfer in the reductase domain of iNOS has previously been determined to be CaMindependent [13]; however, electron transfer from the FMN to the catalytic heme centre in iNOS is CaM dependent $[5,14]$. The iNOS enzyme can only be purified when coexpressed with $\mathrm{CaM}$ and the two proteins remain tightly associated even after the removal of $\mathrm{Ca}^{+2}$. For this reason, $\mathrm{CaM}$ has been classified as a subunit of iNOS [15]. This has made it difficult to dissect the molecular details of the interaction between the iNOS enzyme and CaM. However, we have developed a system for coexpressing human iNOS and $\mathrm{CaM}$ proteins that has allowed for the mapping of the binding of $\mathrm{CaM}$ dependent activation of all three NOS isozymes.

Two aspects of the CaM-NOS interaction that are of fundamental interest are how CaM is able (1) to bind, and (2) to activate the three isoforms of NOS. The major differences between the CaM-regulated isoforms of nNOS/eNOS and the $\mathrm{Ca}^{2+}$ insensitive isoform iNOS are the intrinsic regulatory elements, including a unique autoregulatory insert within the FMN domain of eNOS/nNOS $[16,17]$ and the C-terminal tail $[18,19]$. The removal of the cNOS autoregulatory elements within the FMN domain by mutagenesis have been shown to decrease the $\mathrm{Ca}^{2+}$-dependent $\mathrm{CaM}$ activation and increase the enzyme activities of nNOS and eNOS at lower $\mathrm{Ca}^{2+}$ concentrations $[16,17]$. The removal of the C-terminal tail from the cNOS enzymes resulted in an enzyme with enhanced electron transfer rates through the reductase domain in the absence of $\mathrm{CaM}$; however, the activity of the enzyme was equivalent to wild-type cNOS enzyme in the presence of $\mathrm{Ca}^{+2} / \mathrm{CaM}$ [19]. Similiarly, a truncated Cterminal tail mutant of iNOS demonstrated a 7-10 fold increase in electron transfer rates in its reductase domain when compared to wild-type iNOS [20]. These results suggest that the C-terminal tail extension found in the NOS enzymes is involved in regulating electron transfer through the NOS enzymes.

In a subsequent study, a mutant of nNOS without its autoinhibitory domain and C-terminal tail extension was produced. It was found that $\mathrm{CaM}$ no longer modulates electron transfer through the reductase domain of this nNOS mutant; however, $\mathrm{CaM}$ was still required to promote electron transfer from the FMN domain to the heme for $\cdot \mathrm{NO}$ production [21]. Clearly, the concerted interactions of the autoinhibitory domain and C-terminal tail extension are involved in the binding and regulation of electron transfer within the cNOS enzymes by CaM.
The three NOS isoforms achieve their biological functions via an intricate regulation of interdomain electron transfer (IET) processes [4]. These IET processes are key steps in $\cdot \mathrm{NO}$ synthesis through coupling reactions between the flavins and heme domains. Uncoupled or partially coupled NOS results in the synthesis of reactive oxygen species such as superoxide, hydrogen peroxide, and peroxynitrite [22]. There have been several studies demonstrating that different regions and elements of $\mathrm{CaM}$ play an important role in the binding and activation of the cNOS holoenzymes [10, 23-26]. While eNOS and nNOS demonstrate similarities in their $\mathrm{Ca}^{2+}$-dependence, regulatory elements (autoinhibitory domain and C-terminal tail) and their binding and activation by $\mathrm{CaM}$, there has been very little reported on the specific elements in $\mathrm{CaM}$ that are important for the activation of iNOS. This review summarizes our research findings using a variety of $\mathrm{CaM}$ mutants to map the particular elements in $\mathrm{CaM}$ that are required to bind and activate the NOS isozymes, with a particular focus on the interactions between CaM and iNOS.

\section{Does CaM Binding Affect the Redox Potential of NOS Isozymes?}

One reason why CaM binding to NOS is vital in terms of electron transfer could be due to a CaM-dependent change in the redox potential difference between the flavins and the heme of NOS. This possibility was investigated using both purified domains and holo NOS enzymes. It was shown that $\mathrm{CaM}$ binding to the isolated reductase or oxygenase domains of nNOS does not result in a significant change in the redox potential of the flavin or heme cofactors [27]. Further studies showed $\mathrm{Ca}^{2+} / \mathrm{CaM}$ has no significant effect on the potentials of any of the redox couples in the cNOS enzymes and that the thermodynamics of electron transfer through all three NOS isoforms are basically similar [28]. However, another study reported that the binding of $\mathrm{CaM}$ and NADP+ to the nNOS reductase domain does in fact perturb the redox potential of the flavins [29]. In either case, all of these studies agree that $\mathrm{CaM}$ binding is pivotal for efficient electron transfer to occur in NOS.

\section{Posttranslational Modifications of NOS and their Effects on CaM Binding/Activation}

Posttranslational modifications of the cNOS enzymes, particularly eNOS, have been studied extensively. It has been shown that eNOS can be myristoylated, palmitoylated, acetylated, and phosphorylated in its oxygenase domain [30]. These posttranslational modifications have been implicated in the enzyme's localization in the cell (cytosol, membranes or organelles) but are suggested to have no effect on $\cdot \mathrm{NO}$ production levels, with the exception of phosphorylation [3133]. Several laboratories reported that both nNOS and eNOS contained serine/threonine protein kinase phosphorylation sites in the tail region of the reductase domain, Ser1177 for human eNOS and Ser1412 for rat nNOS, and in the autoinhibitory domain, Ser633 in eNOS and Ser847 in nNOS $[34,35]$. These posttranslational modifications result in modified NOS activity including negative modulation of eNOS activity which was at least partially due to the suppression of $\mathrm{CaM}$ binding.

The iNOS enzyme is also known to undergo posttranslational modifications. These modifications include 
an N-terminal palmitoyl group, which influences the localization of iNOS in the cell [36]. The iNOS enzyme has also been shown to be phosphorylated by tyrosine kinases but the effect of this modification on iNOS activity remains unclear [37]. Lastly, iNOS has been shown to have its rate of turnover in the cell directly regulated by the proteosomal degradation pathway [38]. In order to control $\bullet \mathrm{NO}$ synthesis rates in the cell, the turnover of iNOS must be tightly regulated to avoid oxidative stress and damage. Clearly, posttranslational modifications and protein turnover are involved in the extrinsic regulation of iNOS.

Studying the Affect of Post-Translational Modifications of CaM on the Binding and Activation of the NOS Isozymes

Chemically modified CaM proteins have been used to simulate naturally occurring post-translational modifications including the oxidation of methionines and serine/threonine phosphorylation. In addition, a method commonly used to identify specific amino acids and elements in CaM essential for CaM-dependent enzyme activation is the use of CaM mutant proteins. These mutant proteins have included a variety of single point mutations, the exchange of EF hand motifs within $\mathrm{CaM}$, and truncations of CaM. During our studies, the role of different $\mathrm{CaM}$ residues, elements and regions on aspects of NOS enzymatic function was assessed by spectrophotometric assays that measure three distinct activities associated with NOS: NADPH oxidation, cytochrome $c$ reduction, and nitric oxide $(\bullet \mathrm{NO})$ generation as assessed by the oxyhemoglobin capture assay (Fig. 1).

\section{Oxidation of CaM Methionine Residues}

The oxidation of methionines in CaM occurs during biological aging and it has been reported that essentially all of the isolated CaM from senescent brain is oxidized [39]. $\mathrm{CaM}$ is particularly sensitive to oxidation under conditions of oxidative stress as a result of the large number of surfaceexposed methionines that are involved in target protein recognition and activation [40]. Mutant proteins that permit the oxidation of specific methionines in $\mathrm{CaM}$ were used to investigate the regulation of NOS activity by protein oxidation [41]. While the site-specific oxidation of Met144 and Met145 had essentially no effect on the maximal extent of eNOS activation, these same modifications resulted in a reduction of nNOS activity that was associated with decreased rates of electron transfer within the reductase domain. These results suggest that site-specific oxidative modifications can differentially regulate target protein function and that methionine oxidation can modulate cell signaling under conditions of oxidative stress.

\section{CaM Phosphomimetics and Central Linker Mutations}

The central linker of CaM has been shown to undergo phosphorylation by casein kinase II at threonine 79 and serine 81 [42]. The effect that these posttranslational mutations have on the electrostatic interactions of the central linker on CaM's ability to bind and activate the NOS enzymes was examined. Phosphomimetic CaM proteins were generated by replacing select threonine and serine residues with an aspartic acid. These phosphomimetic proteins fully activated eNOS, but were slightly less effective than unmodified $\mathrm{CaM}$ at activating the other isoforms [43]. Further investigations were performed on the role of the central linker by using a positively-charged central linker and central linker deletion CaM mutants. These investigations demonstrated that the glutamate rich region in the central linker (residues 82-87) plays an important role in the tight and irreversible association of CaM to iNOS.

\section{Roles of CaM EF Hands and Lobes in the Binding and Activation of NOS Isozymes}

As described previously, $\mathrm{CaM}$ is a 148-amino-acid protein consisting of two globular domains joined by a central linker with each domain containing an EF hand pair. The C-terminal EF hand pair has an affinity for $\mathrm{Ca}^{2+}\left(\mathrm{K}_{\mathrm{d}}=\right.$ $\left.10^{-6} \mathrm{M}\right) 10$-fold greater than the $\mathrm{N}$-terminal EF hand pair $\left(\mathrm{K}_{\mathrm{d}}\right.$ $=10^{-5} \mathrm{M}$ ) [40]. The role of the four CaM EF hands in the activation of the three NOS isoforms was investigated by using a series of mutant forms of $\mathrm{CaM}$, including $\mathrm{CaM}$ troponin $\mathrm{C}$ chimeras. The overall structure of troponin $\mathrm{C}$ (TnC) is similar to $\mathrm{CaM}$ since both proteins have two globular domains linked by a central helix with each globular domain composed of two EF hands; however, TnC has only $70 \%$ sequence similarity with $\mathrm{CaM}$ and the first $\mathrm{EF}$ hand of TnC does not bind $\mathrm{Ca}^{2+}[24,26]$.

Replacement of any CaM EF hand by its TnC cognate resulted in significantly decreased $\cdot \mathrm{NO}$ synthesis by cNOS; in contrast with the iNOS results, EF hand 2 was the least sensitive even though it diverges furthest in $\mathrm{TnC}$.

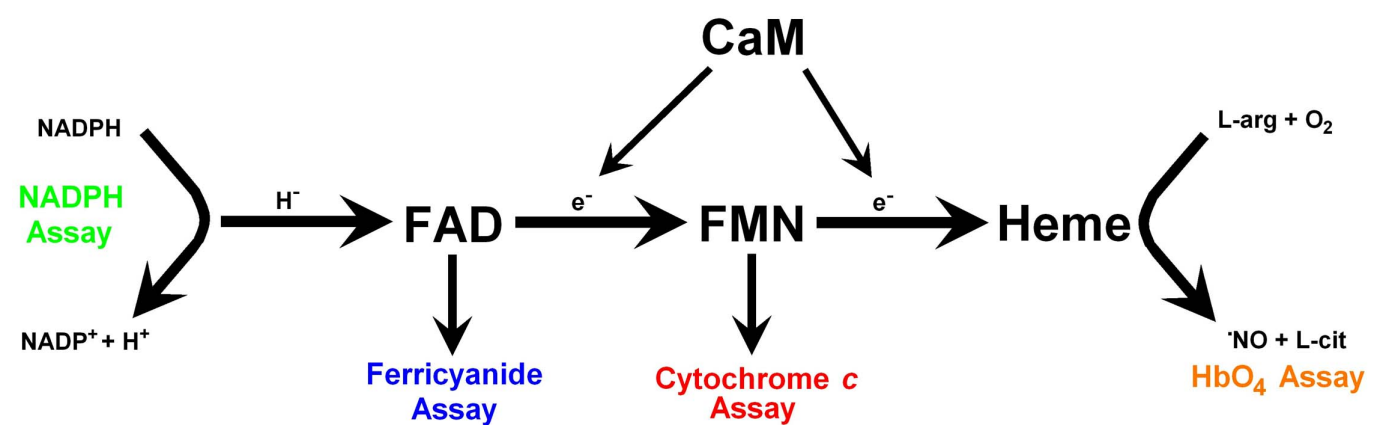

Fig. (1). Spectrophotometric assays employed to monitor electron transfer within the NOS enzymes. (1) The NADPH assay-measures the oxidation of NADPH to NADP ${ }^{+}+\mathrm{H}^{+}$by monitoring decreases in absorbance at $340 \mathrm{~nm}$. (2) The ferricyanide assay-measures the hydride transfer to the FAD by monitoring the reduction of Fe(III) to Fe(II) with decreases in absorbance at $420 \mathrm{~nm}$. (3) The cytochrome c assay measures electron transfer through the reductase domain to the FMN by monitoring the reduction of cytochrome $\mathrm{c}$ with increases in absorbance at $550 \mathrm{~nm}$. (4) The oxyhemoglobin $\left(\mathrm{HbO}_{4}\right)$ capture assay-measures electron transfer to the heme by monitoring the production of $\bullet \mathrm{NO}$ from L-arginine with the conversion of oxy to methemoglobin with increases in absorbance at $401 \mathrm{~nm}$. 
Interactions with the reductase domain are dominant in cNOS activation and EF hand 1 was shown to be critical for the activation of both nNOS and eNOS. In contrast, while all mutations to the any of the four EF hands had no negative effect on cytochrome $c$ reduction by iNOS, domains 2 and 3 of $\mathrm{CaM}$ contain important elements required for the $\mathrm{Ca}^{2+} / \mathrm{CaM}$ independence of $\cdot \mathrm{NO}$ production by the iNOS enzyme [7]. This finding was later confirmed by another study using CaM mutants with glutamate to glutamine mutations at site 12 in each EF hand of CaM [44].

The role of each lobe was investigated by creating CaM mutants with duplication of either the $\mathrm{N}$ - and C-terminal lobes [6]. The results of this investigation indicated that the $\mathrm{N}$-terminal EF hand pair of CaM contains important binding and activating elements for iNOS, whereas the N-terminal EF hand pair in conjunction with the central linker region is required for cNOS enzyme binding and activation. Subsequent investigations of the effect of calcium binding to each of the two lobes were performed using mutant CaM proteins that were no longer able to bind their full complement of $\mathrm{Ca}^{2+}$. CaM with mutations of glutamate to glutamine residues at position 12 of each $\mathrm{EF}$ hand in $\mathrm{CaM}$ (-Z co-ordination site in a typical EF hand) have been used to study specific $\mathrm{Ca}^{2+}$-binding sites in $\mathrm{CaM}$ and its association to nNOS [10]. Our investigation using CaM-TnC chimeric proteins found that CaM EF hand 1 binding to $\mathrm{Ca}^{2+}$ was crucial for nNOS activity. These investigations were expanded by using $\mathrm{CaM}$ mutants that were deficient in binding $\mathrm{Ca}^{2+}$ with mutations in the $\mathrm{N}$-lobe $\left(\mathrm{CaM}_{12}\right)$, the $\mathrm{C}$ lobe $\left(\mathrm{CaM}_{34}\right)$, or both lobes of $\mathrm{CaM}\left(\mathrm{CaM}_{1234}\right)$ to determine their effect on the binding and activation of the $\mathrm{Ca}^{2+}$ dependent nNOS and $\mathrm{Ca}^{2+}$-independent iNOS isoforms [45]. These studies were performed in order to unequivocally determine whether the binding and activation of iNOS by $\mathrm{CaM}$ is $\mathrm{Ca}^{2+}$-independent by using $\mathrm{CaM}_{1234}$ to emulate $\mathrm{Ca}^{2+}$ free $\mathrm{CaM}$; however, as previous studies have demonstrated that the $\mathrm{N}$ - and C-terminal lobes of CaM can also be devoid of $\mathrm{Ca}^{2+}$ when associated with its target proteins [46,47], $\mathrm{CaM}_{12}$ and $\mathrm{CaM}_{34}$ were used to determine if the $\mathrm{Ca}^{2+}$ independent association of CaM to iNOS is related solely to the $\mathrm{Ca}^{2+}$-free $\mathrm{N}$ - or C-terminal lobe of CaM. These investigations showed that apo- $\mathrm{CaM}$ is capable of binding and partially activating iNOS in a $\mathrm{Ca}^{2+}$-independent manner, however, $\mathrm{Ca}^{2+}$-replete conditions are required for the full activation of the enzyme. Upon further analysis using CD and fluorescence, we determined that the C-terminal lobe of $\mathrm{CaM}$ is responsible for the binding and stability of iNOS, while the $\mathrm{N}$-terminal domain contains the important elements requiring $\mathrm{Ca}^{2+}$-replete conditions for the full activation of iNOS [6]. This is consistent with previous studies that demonstrated there is some $\mathrm{Ca}^{2+}$-dependence in the synthesis of $\cdot \mathrm{NO}$ by iNOS $[6,7,48]$.

\section{Structural Basis of NOS Isoform Specific CaM-Binding and Activation Properties}

There is considerable interest in understanding the structural basis of CaM's target protein interactions and diverse regulatory functions. It is well established that CaM is able to interact with its target enzymes in many different conformations, varying from being tightly wrapped when bound to myosin light chain kinase [49], and eNOS [50], as determined by NMR and x-ray crystallography, to having an extended structure when bound to the edema factor of adenylyl cyclase from Bacillus anthacis, as determined by FRET [51] and x-ray crystallography [52]. The fact that the FRET results of CaM bound to the edema factor were later supported by X-ray crystallography confirms that the conformation of CaM determined by FRET is representative of a protein's true conformation in vitro. The structure of $\mathrm{CaM}$ bound to a peptide corresponding to the CaM-binding domain of eNOS [50] and nNOS (PDB 2060) have been solved and it is known that CaM binds to the cNOS enzymes in an anti-parallel orientation [50,53]. We used FRET studies to show that the iNOS also binds to CaM in an anti-paralllel orientation [54] and a collapsed conformation when bound to an iNOS CaM-binding domain peptide [55]. Our findings were corroborated by the recently published structure of CaM bound human iNOS FMN domain [56].

\section{Biophysical Studies to Characterize CaM Bound to the NOS Isozymes}

Although the structures and orientation of CaM bound to NOS peptides have been determined (Fig. 2), the conformation of $\mathrm{CaM}$ when bound to the holo-NOS enzymes has remained elusive. This is due in large part to the size of the NOS enzymes $(\sim 124-160 \mathrm{kDa})$, which would not make it amenable to determine their structures using NMR, as well as the dynamic structural rearrangements that occur within the reductase domain and CaM-binding domains of the NOS enzymes, which would make it difficult to obtain the protein crystals required to perform $\mathrm{x}$-ray crystallography. There are numerous solved crystal structures of the oxygenase domains for each of the NOS isozymes, as well as a solved crystal structure of the nNOS reductase domain without the autoinhibitory domain and CaM-binding domain being shown in the structure due to their highly flexible nature (Fig. 2, [56,57]).

Although there are numerous crystal structures of various regions of the NOS enzymes, an intact model of the full length NOS enzyme has remained elusive. Piecing together the heme and reductase domain structures by computational docking studies may provide some insights [56], but alternative models will be difficult to distinguish when compared to the favored ones.

One control mechanism proposed for electron transfer in the reductase domain of nNOS is the NADPH conformational lock [58,59]. Previous studies have suggested that this "locked" conformation occurs after NADPH transfers a hydride to FAD to form $\mathrm{FADH}_{2}$ and a subsequent NADPH molecule binds and is unable to transfer its hydride to reduced $\mathrm{FADH}_{2}$. In order to unlock the reductase domain, the FAD needs to be regenerated to accept another hydride from NADPH and pass electrons through the rest of the NOS enzyme. The large conformational change that CaM induces in the reductase domain of the NOS enzymes allows for the FMN domain to interact with both the FAD to accept electrons and pass the electrons on to the heme during catalysis. The electron transfer from $\mathrm{FADH}_{2}$ to FMN regenerates FAD thereby unlocking the reductase domain [58,59]. Clearly, these conformational changes caused by $\mathrm{CaM}$ are important in stimulating efficient electron transfer within the NOS enzymes. 


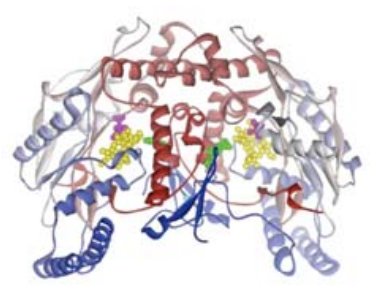

a

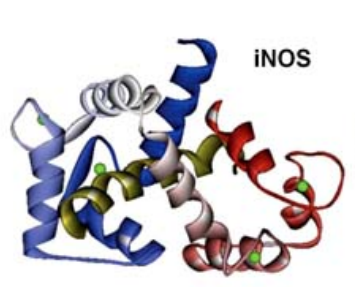

d

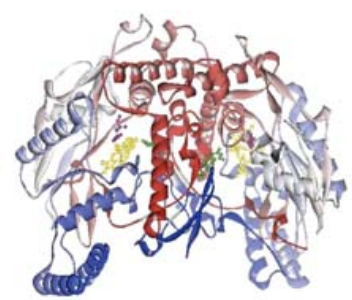

b

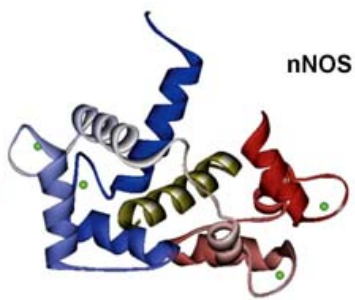

e

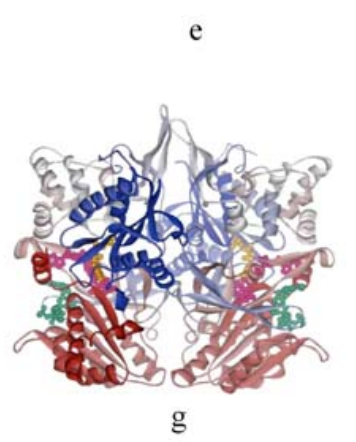

g

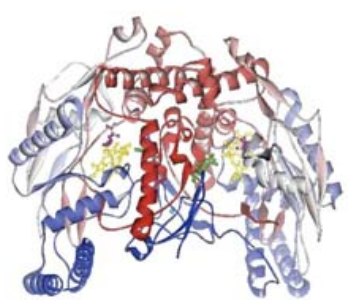

$\mathrm{c}$

Fig. (2). Solved crystal structures of the oxygenase, CaM-binding and reductase domains of NOS. The crystal structure of (a) iNOS, (b) nNOS and (c) eNOS oxygenase domain dimer containing co-factors heme and $\mathrm{H}_{4} \mathrm{~B}$, and L-arginine represented in yellow, green and pink ball and stick figures, respectively. The cyan sphere between the dimers is the $\mathrm{Zn}^{2+}$ ion. (d)-(f) Comparison of the CaM binding region of the three isoforms (gold) in complex with CaM (blue and red which represent N- and C-terminal lobes, respectively). The cNOS enzymes are very similar whereas iNOS has CaM more tightly wrapped around the peptide, especially in the N-terminal region of CaM's central linker. The central linker region of $\mathrm{CaM}$ is also not as extended in the iNOS-CaM structure as in the cNOS isoforms. (g) nNOS reductase domain with the cofactors NADPH, FAD and FMN represented in teal, magenta and orange, respectively. Models were derived from PDB 3E65 (a), 1FOP (b), 1ZVL (c), 1NIW (d), $2 \mathrm{O} 60$ (e), 3HR4 (f), and 1TLL (g). Structures were visualized using Accelrys DS visualize 2.5.

Protein modeling has demonstrated that the distances between the FAD and FMN cofactors in the reductase domain are too large for electron transfer to take place and the distances between the FMN and heme centres are greater than $25 \AA$ [5]. These distances are too large for proper electron transfer to occur from the flavins to the heme; therefore it is probable that a major structural rearrangement is necessary for the NOS enzymes to properly function. This has led to the suggestion that $\mathrm{CaM}$ facilitates large conformational changes within NOS by moving the FMNbinding domain, (a region of the reductase domain that may be highly dynamic and flexible due to a hinge domain) from an electron-accepting position (FAD-FMN) and moving to an electron donating position (FMN-heme). This has led to the proposal of an "FMN-domain tethered shuttle" model [5]. This model involves the swinging of the FMN domain from its original electron-accepting (input) state to a new electron-donating (output) state that would facilitate efficient IET between the FMN and the catalytic heme in the oxygenase domain. Without a complete structure of the holoenzyme, it is difficult to envisage how this takes place. Crystallographic and mutagenesis investigations indicated that the most likely regions of interaction are an acidic cluster of residues on the surface of the FMN domain and a basic patch of residues on the surface of the oxygenase domain [60-62]. The contact regions in the input and output states appear to be controlled by electrostatic interactions.

The structure of the functional output state has not yet been determined but the complex between $\mathrm{CaM}$ and the FMN domain of human iNOS has recently been reported (Fig. 3, [56]). This complex has been crystallized in four different conformations showing the flexible nature of the interdomain interactions. Edge to edge distances of 13 to 18 $\AA$ between the heme iron of one subunit and the FMN center of the other subunit of the iNOS dimer were recently determined using pulse EPR experiments [63]. These distances are in excellent agreement with IET kinetic studies [64,65], low temperature MCD data [66], and support the tethered shuttle model. 

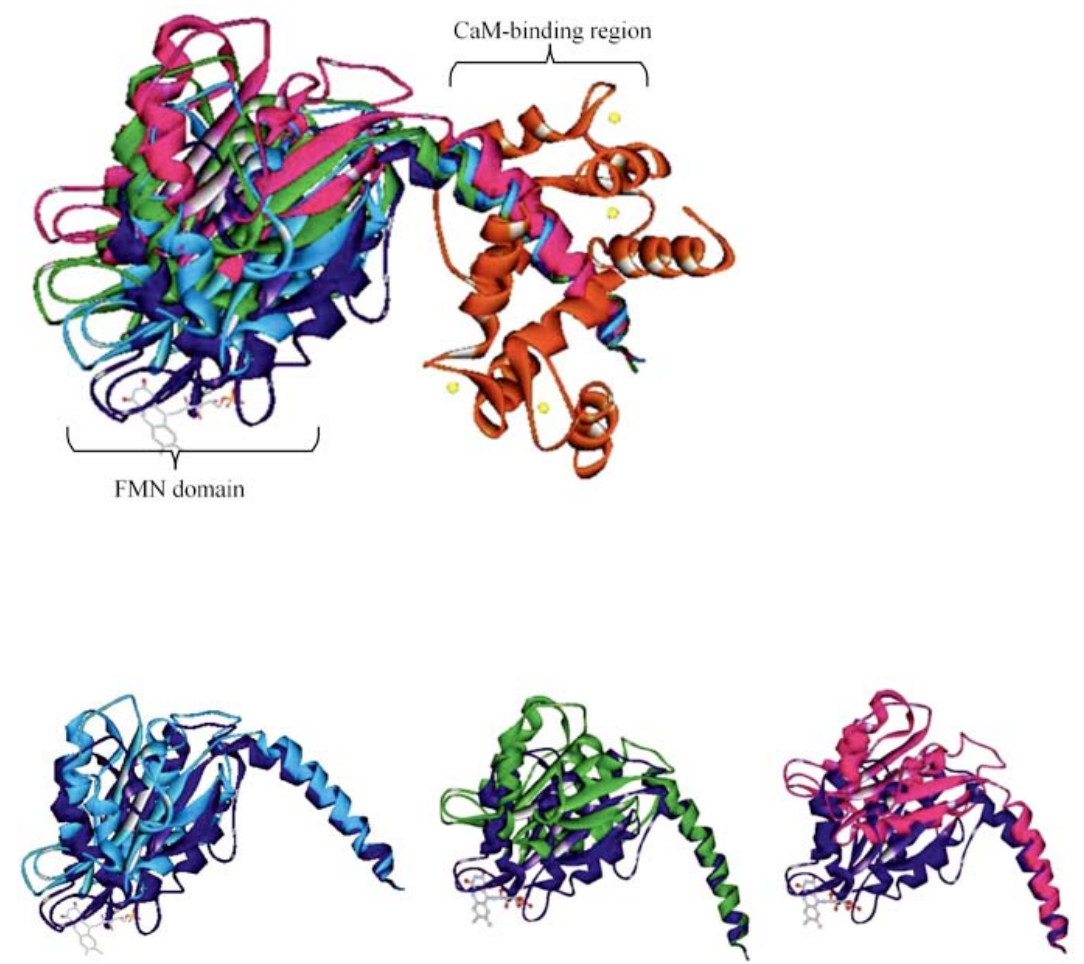

Fig. (3). Crystal structures of CaM bound to the iNOS FMN domain with the CaM binding region. The four structures solved by Xia et al [56] were superimposed by aligning the CaM binding domain to show the range of motion of the FMN domain. Panel A shows all four crystal structures superimposed with $\mathrm{Ca}^{2+}$ (yellow sphere)-bound $\mathrm{CaM}$ (orange) wrapped around the iNOS CaM-binding region. Panels $\mathrm{B}$, C and D demonstrate the movement of each structure with respect to the purple structure with the bound CaM removed. Panel B shows the least amount of movement while Panel D shows the largest. The FMN cofactor of the purple structure is represented in a ball and stick format. Structures were derived from PDB 3HR4 and visualized using Accelrys DS visualize 2.5.

The holoenzyme could essentially be described as a molecular machine with moving parts and it is therefore not surprising that a crystal structure of the full complex has not been reported. The enzyme lacks a unique structure; there are instead many conformations, including a well defined FMN-FAD-NADPH domain structure with a disoriented oxygenase domain, and a well defined, if transient, FMNoxygenase domain structure, with no well defined orientation with respect to the FAD-NADPH unit.

\section{Model of the CaM-Dependent Regulation of iNOS}

Our current understanding of the CaM-dependent regulation of iNOS builds upon the previous model proposed by Garcin and coworkers for the $\mathrm{Ca}^{2+}$-dependent activation of the cNOS enzymes [57]. Since previous studies have shown that electron transfer in the reductase domain of iNOS is CaM-independent [13], the only point of electron transfer that appears to be directly affected by CaM is the FMN to heme electron transfer step. We propose a model for the CaM-dependent regulation of iNOS that is based upon a previous study [57] with the exception that the reductase domain is not shown as a dimer (see Fig. 4). Although this model is simplistic in describing the effect of the various domains of $\mathrm{CaM}$ on electron transfer with iNOS, it does demonstrate the importance of $\mathrm{Ca}^{2+}$ binding to the $\mathrm{C}$ terminal domain of $\mathrm{CaM}$ to promote efficient electron transfer from the FMN domain to the heme for $\cdot \mathrm{NO}$ synthesis. Each monomer of iNOS is shown in white and grey, respectively. The structural zinc at the dimer interface of the oxygenase domain is shown in yellow. The N- and C- terminal domains of $\mathrm{CaM}$ are shown in red and blue, respectively. $\mathrm{Ca}^{2+}$-deplete and $\mathrm{Ca}^{2+}$-replete $\mathrm{EF}$ hands of $\mathrm{CaM}$ are shown as white and green circles. CaM binds in an antiparallel fashion with the N-terminal domain of $\mathrm{CaM}$ binding closer to the reductase domain and interacts directly with the FMN domain. The C-terminal domain of CaM binds closer to the oxygenase domain. Our studies have shown that the $\mathrm{N}$-terminal domain of $\mathrm{CaM}$ contains important binding and activating elements for iNOS [6]. At basal $\mathrm{Ca}^{2+}$ concentrations, this $\mathrm{CaM}$ domain is $\mathrm{Ca}^{2+}$-deplete and binds very tightly to the N-type site of the iNOS CaM-binding domain. The N-terminal domain of CaM does not appear to have any $\mathrm{Ca}^{2+}$-dependent effect on electron transfer rates within iNOS $[45,54]$. (A) Apo-CaM is able to bind to the iNOS enzyme. The reductase domain of iNOS is CaMindependent and is able to transfer electrons efficiently from NADPH through the reductase domain to the FMN [13]. After electrons are transferred to the FMN, a CaM-dependent conformational change in the FMN domain occurs, where the FMN domain moves from an electron accepting position to an electron donating position. The electrons from $\mathrm{FMNH}_{2}$ are able to transferred to the catalytic heme of the other NOS monomer; however, this structural change in the reductase domain is not perfect $\left(\sim 70 \%\right.$ when compared to $\mathrm{Ca}^{2+}$-replete $\mathrm{CaM}$ ) and electrons are able to be uncoupled from $\cdot \mathrm{NO}$ production to produce superoxide radicals $\left(\cdot \mathrm{O}_{2}{ }^{-}\right)$. The production of $\bullet^{-}{ }_{2}^{-}$has previously been linked to electron uncoupling from the flavins in the reductase domain of iNOS [56]. This uncoupling, which results in an NADPH oxidized to $\bullet \mathrm{NO}$ produced ratio greater than $2: 1$, as well as lower $\bullet \mathrm{NO}$ 

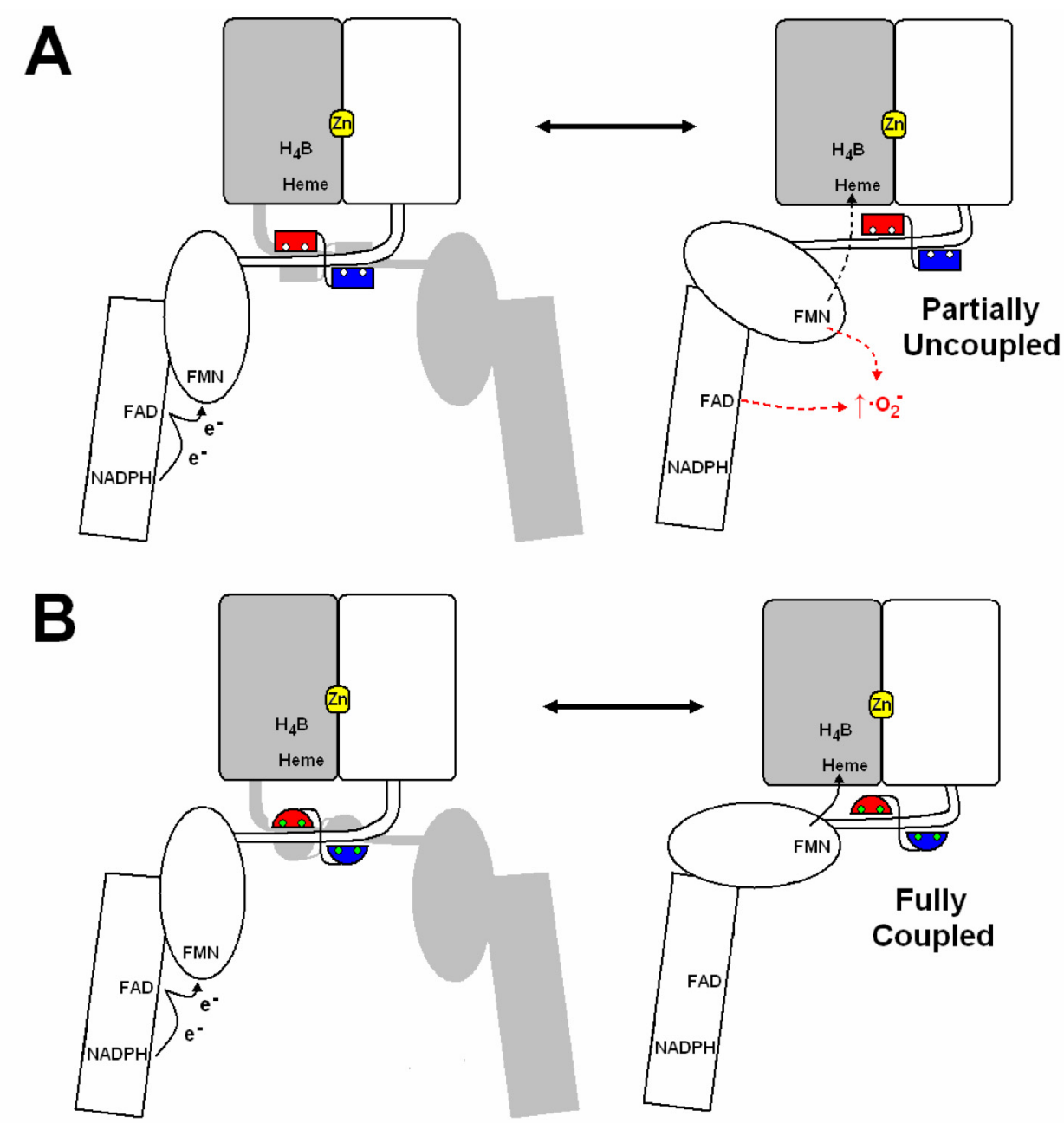

Fig. (4). Model for CaM-dependent regulation of •NO by iNOS. This model is based upon a previous study [57] with the exception that the reductase domain is not shown as a dimer (see text for explanation).

production rates by iNOS has been attributed to the $\mathrm{Ca}^{2+}$ deplete $\mathrm{C}$-terminal lobe of $\mathrm{CaM}$, as previously reported [45, 54]. (B) An increase in free $\mathrm{Ca}^{2+}$ concentration causes the Cterminal domain of $\mathrm{CaM}$ to become $\mathrm{Ca}^{2+}$-replete. This directly affects the CaM-dependent conformational change in the FMN domain to dock perfectly with the oxygenase domain so that it can pass its electons efficiently to the catalytic heme. This results in the reductase domain derived electrons being tightly coupled to $\cdot \mathrm{NO}$ production with an NADPH oxidation to $\cdot \mathrm{NO}$ produced ratio of $1.5: 1$. Although it is still unclear if the N-terminal domain of $\mathrm{CaM}$ is $\mathrm{Ca}^{2+}$ deplete or $\mathrm{Ca}^{2+}$-replete in the fully active form of iNOS, it is clear that $\mathrm{Ca}^{2+}$ binding to $\mathrm{CaM}$ is required for electrons to be fully coupled to $\bullet \mathrm{NO}$ synthesis.

\section{CONCLUSIONS}

Due to the biological importance of $\bullet \mathrm{NO}$ in many different inter- and intracellular processes, understanding how these enzymes work is of significant interest to the biochemical and medical community. Since all three NOS enzymes have shown inherent differences in their binding and activation by $\mathrm{CaM}$, especially in their $\mathrm{Ca}^{2+}$-dependence or -independence, our laboratory set about deciphering the exact regulatory elements present in $\mathrm{CaM}$ that are directly involved in regulating the NOS enzymes. Through the use of an assortment of CaM mutant proteins, we have mapped the sites in CaM responsible for the binding and activation of the NOS enzymes using a variety biophysical techniques including kinetic analyses and fluorescence. Our investigation of CaM-NOS interactions has resulted in many significant milestones. These included using a co-expression method for iNOS with various CaM mutants that enabled us to be the first to ever report on specific elements in $\mathrm{CaM}$ required for the activation of iNOS. We were also the first to monitor through the use of fluorescence and FRET the dynamic conformational changes in $\mathrm{CaM}$ when associated to NOS peptides and enzymes in the presence of increasing free $\mathrm{Ca}^{2+}$ concentrations. By combining the cumulative results from our studies on CaM-iNOS interactions, we were able to propose a strong working model for the CaM-dependent regulation of iNOS. The dynamic properties of $\mathrm{CaM}$ and the conformational changes that $\mathrm{CaM}$ causes in the NOS enzymes is a complex, and at the same time, a very interesting protein-protein interaction model system. Future structural studies working towards a full length NOS structure will hopefully clarify how the binding of $\mathrm{CaM}$ causes global conformational changes in the NOS enzymes to initiate $\bullet \mathrm{NO}$ synthesis. The results reported in this review 
have expanded and improved the present understanding of the CaM-dependent binding and activation of the NOS isozymes, particularly the interactions between $\mathrm{CaM}$ and iNOS.

\section{ACKNOWLEDGEMENTS}

This work was supported by NSERC Grant 183521 (to J.G.G). D.E.S. is the grateful recipient of an Ontario Graduate Scholarship and a UW President's Scholarship.

\section{CONFLICT OF INTERESTS}

None.

\section{SUPPLEMENTARY MATERIAL}

Supplementary material is available on the publishers Web site along with the published article.

\section{REFERENCES}

[1] Ikura, M.; Ames, J.B. Genetic polymorphism and protein conformational plasticity in the calmodulin superfamily: two ways to promote multifunctionality. Proc. Natl. Acad. Sci. USA, 2006, 103, 1159-1164.

[2] Persechini, A.; Kretsinger, R.H. The central helix of calmodulin functions as a flexible tether. J. Biol. Chem., 1988 263, 1217512178.

[3] Alderton, W.K.; Cooper, C.E.; Knowles, R.G. Nitric oxide synthases: structure, function and inhibition. Biochem J., 2001, 357, 593-615.

[4] Roman, L.J.; Martasek, P.; Masters, B.S. Intrinsic and extrinsic modulation of nitric oxide synthase activity. Chem. Rev., 2002,102, 1179-1190.

[5] Ghosh, D.K.; Salerno, J.C. Nitric oxide synthases: domain structure and alignment in enzyme function and control. Front. Biosci., 2003, 8, d193-d209.

[6] Spratt, D.E.; Newman, E.; Mosher, J.; Ghosh, D.K.; Salerno, J.C.; Guillemette, J.G. Binding and activation of nitric oxide synthase isozymes by calmodulin EF hand pairs. FEBS J., 2006, 273, 17591771.

[7] Newman, E.; Spratt, D.E.; Mosher, J.; Cheyne, B.; Montgomery, H.J.; Wilson, D.L.; Weinberg, J.B.; Smith, S.M.; Salerno, J.C.; Ghosh, D.K.; Guillemette, J.G. Differential activation of nitricoxide synthase isozymes by calmodulin-troponin $\mathrm{C}$ chimeras. $J$. Biol. Chem., 2004, 279, 33547-33557.

Sagami, I.; Daff, S.; Shimizu, T. Intra-subunit and inter-subunit electrontransfer in neuronal nitric-oxide synthase: effect of calmodulin on heterodimer catalysis. J. Bio.l Chem., 2001, 276, 30036-30042.

[9] Siddhanta, U.; Presta, A.; Fan, B.; Wolan, D.; Rousseau, D.L.; Stuehr, D.J. Domain swapping in inducible nitric-oxide synthase. Electron transfer occurs between flavin and heme groups located on adjacent subunits in the dimer. J. Biol. Chem., 1998, 273, 18950-18958.

[10] Stevens-Truss, R.; Beckingham, K.; Marletta, M.A. Calcium binding sites of calmodulin and electron transfer by neuronal nitric oxide synthase. Biochemistry, 1997, 36, 12337-12345.

[11] Chen, P.F.; Tsai, A.L.; Berka, V.; Wu, K.K. Endothelial nitricoxide synthase. Evidence for bidomain structure and successful reconstitution of catalytic activity from two separate domains generated by a baculovirus expression system. J. Biol. Chem., 1996, 271, 14631-14635.

[12] Abu-Soud, H.M.; Yoho, L.L.; Stuehr, D.J. Calmodulin controls neuronal nitric-oxide synthase by a dual mechanism. Activation of intra- and interdomain electron transfer. J. Biol. Chem., 1994, 269, 32047-32050.

[13] Newton, D.C.; Montgomery, H.J.; Guillemette, J.G. The reductase domain of the human inducible nitric oxide synthase is fully active in the absence of bound calmodulin. Arch. Biochem. Biophys., 1998, 359, 249-257.

[14] Feng, C.; Thomas, C.; Holliday, M.A.; Tollin, G.; Salerno, J.C.; Ghosh, D.K.; Enemark, J.H. Direct measurement by laser flash photolysis of intramolecular electron transfer in a two-domain construct of murine inducible nitric oxide synthase. J. Am. Chem. Soc., 2006, 128, 3808-3811.

[15] Cho, H.J.; Xie, Q.W.; Calaycay, J.; Mumford, R.A.; Swiderek, K.M.; Lee, T.D.; Nathan, C. Calmodulin is a subunit of nitric oxide synthase from macrophages. J. Exp. Med., 1992, 176, 599-604.

[16] Daff, S.; Sagami, I.; Shimizu, T. The 42-amino acid insert in the FMN domain of neuronal nitric-oxide synthase exerts control over $\mathrm{Ca}^{2+} /$ calmodulin-dependent electron transfer. J. Biol. Chem., 1999, 274, 30589-30595.

[17] Montgomery, H.J.; Romanov, V.; Guillemette, J.G. Removal of a putative inhibitory element reduces the calcium-dependent calmodulin activation of neuronal nitric-oxide synthase. J. Biol. Chem., 2000, 275, 5052-5058.

[18] Harris, D.E.; Roman, L.J.; Martasek, P.; Shea, T.M.; Masters, B.S.S. Regulation of the constitutive nitric oxide synthases through their reductase domains. Biochemistry, 2001, 40, 225-233.

[19] Roman, L.J.; Martasek, P.; Miller, R.T.; Harris, D.E.; De La Garza M.A.; Shea, T.M.; Kim, J.J.; Masters, B.S. The C termini of constitutive nitric-oxide synthases control electron flow through the flavin and heme domains and affect modulation by calmodulin. $J$. Biol. Chem., 2000, 275, 29225-29232.

[20] Roman, L.J., Miller, R.T., De La Garza, M.A., Kim, J.J. and Siler Masters, B.S. The $\mathrm{C}$ terminus of mouse macrophage inducible nitric-oxide synthase attenuates electron flow through the flavin domain. J Biol Chem., 2000, 275, 21914-21919.

[21] Roman, L.J.; Masters, B.S. Electron transfer by neuronal nitricoxide synthase is regulated by concerted interaction of calmodulin and two intrinsic regulatory elements. J. Biol. Chem., 2006,281, 23111-23118.

[22] Chen, C. A.; Druhan, L. J.; Varadharaj, S.; Chen, Y. R.; Zweier, J L. Phosphorylation of endothelial nitric-oxide synthase regulates superoxide generation from the enzyme. J. Biol. Chem., 2008, 283, 27038-27047.

[23] Persechini, A.; Mcmillan, K.; Leakey, P. Activation of myosin light chain kinase and nitric oxide synthase activities by calmodulin fragments. J. Biol. Chem., 1994, 269, 16148-16154.

[24] Su, Z.; Blazing, M.A.; Fan, D.; George, S.E. The calmodulin-nitric oxide synthase interaction: critical role of the calmodulin latch domain in enzyme activation. J. Biol. Chem., 1995, 270, 29117 29122.

[25] Persechini, A.; Gansz, K.J.; Paresi, R.J. Activation of myosin light chain kinase and nitric oxide synthase activities by engineered calmodulins with duplicated or exchanged EF hand pairs. Biochemistry, 1996, 35, 224-228.

[26] Gachhui, R.; Abu-Soud, H.M.; Ghosha, D.K.; Presta, A.; Blazing, M.A.; Mayer, B.; George, S.E.; Stuehr, D.J. Neuronal nitric-oxide synthase interaction with calmodulin-troponin C chimeras. J. Biol. Chem., 1998, 273, 5451-5454.

[27] Noble, M.A.; Munro, A.W.; Rivers, S.L.; Robledo, L.; Daff, S.N.; Yellowlees, L.J.; Shimizu, T.; Sagami, I.; Guillemette, J.G.; Chapman, S.K. Potentiometric analysis of the flavin cofactors of neuronal nitric oxide synthase. Biochemistry, 1999, 38, 1641316418 .

[28] Gao, Y.T.; Smith, S.M.; Weinberg, J.B.; Montgomery, H.J.; Newman, E.; Guillemette, J.G.; Ghosh, D.K.; Roman, L.J.; Martasek, P.; Salerno, J.C. Thermodynamics of oxidation reduction reactions in mammalian nitric-oxide synthase isoforms. J. Biol. Chem., 2004, 279, 18759-18766.

[29] Dunford, A.J.; Rigby, S.E.; Hay, S.; Munro, A.W.; Scrutton, N.S Conformational and thermodynamic control of electron transfer in neuronal nitric oxide synthase. Biochemistry, 2007, 46, 5018-5029.

[30] Benaim, G.; Villalobo, A. Phosphorylation of calmodulin: functional implications. Eur. J. Biochem., 2002, 269, 3619-3631.

[31] Liu, J.; Hughes, T.E.; Sessa, W.C. The first 35 amino acids and fatty acylation sites determine the molecular targeting of endothelial nitric oxide synthase into the Golgi region of cells: a green fluorescent protein study. J. Cell. Biol., 1997, 137, 15251535 .

[32] Michel, T.; Feron, O. Nitric oxide synthases: which, where, how, and why? J. Clin. Invest., 1997,100, 2146-2152.

[33] Hemmens, B.; Mayer, B. Enzymology of nitric oxide synthases. Methods Mol. Biol., 1998, 100, 1-32.

[34] Tran, Q.K.; Leonard, J.; Black, D.J.; Nadeau, O.W.; Boulatnikov, I.G.; Persechini, A. Effects of combined phosphorylation at Ser617 and Ser-1179 in endothelial nitric-oxide synthase on 
$\mathrm{EC}_{50}\left(\mathrm{Ca}^{2+}\right)$ values for calmodulin binding and enzyme activation. $J$. Biol. Chem., 2009, 284, 11892-11899.

[35] Tran, Q.K.; Leonard, J.; Black, D.J.; Persechini, A. Phosphorylation within an autoinhibitory domain in endothelial nitric oxide synthase reduces the $\mathrm{Ca}^{2+}$ concentrations required for calmodulin to bind and activate the enzyme. Biochemistry, 2008, 47, 7557-7566.

[36] Navarro-Lerida, I.; Corvi, M.M.; Barrientos, A.A.; Gavilanes, F.; Berthiaume, L.G.; Rodriguez-Crespo, I. Palmitoylation of inducible nitric-oxide synthase at Cys-3 is required for proper intracellular traffic and nitric oxide synthesis. J. Biol. Chem., 2004, 279, 5568255689.

[37] Pan, J.; Burgher, K.L.; Szczepanik, A.M.; Ringheim, G.E. Tyrosine phosphorylation of inducible nitric oxide synthase: implications for potential post-translational regulation. Biochem. J., 1996, 314, 889894.

[38] Musial, A.; Eissa, N.T. Inducible nitric-oxide synthase is regulated by the proteasome degradation pathway. J. Biol. Chem., 2001, 276, 24268-24273.

[39] Gao, J.; Yin, D.; Yao, Y.; Williams, T.D.; Squier, T.C. Progressive decline in the ability of calmodulin isolated from aged brain to activate the plasma membrane Ca-ATPase. Biochemistry, 1998, 37, 9536-9548.

[40] Crivici, A.; Ikura, M. Molecular and structural basis of target recognition by calmodulin. Annu. Rev. Biophys. Biomol. Struct. 1995, 24, 85-116.

[41] Montgomery, H.J.; Bartlett, R.; Perdicakis, B.; Jervis, E.; Squier, T.C.; Guillemette, J.G. Activation of constitutive nitric oxide synthases by oxidized calmodulin mutants. Biochemistry, 2003, 42, 7759-7768.

[42] Quadroni, M.; James, P.; Carafoli, E. Isolation of phosphorylated calmodulin from rat liver and identification of the in vivo phosphorylation sites. J. Biol. Chem., 1994, 269, 16116-16122.

[43] Spratt, D. E.; Israel, O. K.; Taiakina, V.; Guillemette, J. G. Regulation of mammalian nitric oxide synthases by electrostatic interactions in the linker region of calmodulin. Biochim. Biophys. Acta, 2008, 1784, 2065-2070.

[44] Gribovskaja, I.; Brownlow, K.C.; Dennis, S.J.; Rosko, A.J.; Marletta, M.A.; Stevens-Truss, R. Calcium-binding sites of calmodulin and electron transfer by inducible nitric oxide synthase. Biochemistry, 2005, 44, 7593-7601.

[45] Spratt, D.E.; Taiakina, V.; Guillemette, J.G. Calcium-deficient calmodulin binding and activation of neuronal and inducible nitric oxide synthases. Biochim. Biophys. Acta, 2007, 1774, 1351-1358.

[46] Schumacher, M.A.; Rivard, A.F.; Bachinger, H.P.; Adelman, J.P. Structure of the gating domain of a $\mathrm{Ca}^{2+}$-activated $\mathrm{K}^{+}$channel complexed with $\mathrm{Ca}^{2+} /$ calmodulin. Nature, 2001, 410, 1120-1124.

[47] Shen, Y.; Zhukovskaya, N.L.; Guo, Q.; Florian, J.; Tang, W.J. Calcium-independent calmodulin binding and two-metal-ion catalytic mechanism of anthrax edema factor. EMBO J., 2005, 24, 929-941.

[48] Venema, R.C.; Sayegh, H.S.; Kent, J.D.; Harrison, D.G. Identification, characterization, and comparison of the calmodulinbinding domains of the endothelial and inducible nitric oxide synthases. J. Biol. Chem., 1996, 271, 6435-6440.

[49] Ikura, M.; Clore, G.M.; Gronenborn, A.M.; Zhu, G.; Klee, C.B.; Bax, A. Solution structure of a calmodulin-target peptide complex by multidimensional NMR. Science, 1992, 256, 632-638.

[50] Aoyagi, M.; Arvai, A.S.; Tainer, J.A.; Getzoff, E.D. Structural basis for endothelial nitric oxide synthase binding to calmodulin. EMBO J., 2003, 22, 766-775.

[51] Drum, C.L.; Yan, S.Z.; Sarac, R.; Mabuchi, Y.; Beckingham, K.; Bohm, A.; Grabarek, Z.; Tang, W.J. An extended conformation of calmodulin induces interactions between the structural domains of adenylyl cyclase from Bacillus anthracis to promote catalysis. $J$. Biol. Chem., 2000, 275, 36334-36340.

[52] Drum, C.L.; Yan, S.Z.; Bard, J.; Shen, Y.Q.; Lu, D.; Soelaiman, S.; Grabarek, Z.; Bohm, A.; Tang, W.J. Structural basis for the activation of anthrax adenylyl cyclase exotoxin by calmodulin. Nature, 2002, 415, 396-402.

[53] Zhang, M.; Yuan, T.; Aramini, J.M.; Vogel, H.J. Interaction of calmodulin with its binding domain of rat cerebellar nitric oxide synthase, a multinuclear NMR study. J. Biol. Chem., 1995, 270, 20901-20907.

[54] Spratt, D.E.; Taiakina, V.; Palmer, M.; Guillemette, J.G. Differential binding of calmodulin domains to constitutive and inducible nitric oxide synthase enzymes. Biochemistry, 2007, 46, 8288-8300.

[55] Spratt, D.E.; Taiakina. V.; Palmer, M.; Guillemette, J.G. FRET conformational analysis of calmodulin binding to nitric oxide synthase peptides and enzymes. Biochemistry, 2008, 47, 1200612017.

[56] Xia, C.; Misra, I.; Iyanagi, T.; Kim, J. J. Regulation of interdomain interactions by calmodulin in inducible nitric-oxide synthase. $J$. Biol. Chem., 2009, 284, 30708-30717.

[57] Garcin, E.D.; Bruns, C.M.; Lloyd, S.J.; Hosfield, D.J.; Tiso, M.; Gachhui, R.; Stuehr, D.J.; Tainer, J.A.; Getzoff, E.D. Structural basis for isozyme-specific regulation of electron transfer in nitricoxide synthase. J. Biol. Chem., 2004, 279, 37918-37927.

[58] Craig, D.H., Chapman, S.K. and Daff, S. Calmodulin activates electron transfer through neuronal nitric-oxide synthase reductase domain by releasing an NADPH- dependent conformational lock. J. Biol. Chem., 2002, 277, 33987-33994.

[59] Daff, S. Calmodulin-dependent regulation of mammalian nitric oxide synthase. Biochem Soc Trans., 2003, 31, 502-505.

[60] Panda, K.; Haque, M.M.; Garcin-Hosfield, E.D.; Durra, D.; Getzoff, E.D.; Stuehr, D.J. Surface charge interactions of the FMN module govern catalysis by nitric oxide synthase, J. Biol. Chem., 2006, 281, 36819-36827.

[61] Crane, B.R.; Arvai, A.S.; Gachhui, R.; Wu, C.Q.; Ghosh, D.K.; Getzoff, E.D.; Stuehr, D.J.; Tainer, J.A. The structure of nitric oxide synthase oxygenase domain and inhibitor complexes. Science, 1997, 278, 425- 431.

[62] Shimanuki, T.; Sato, H.; Daff, S.; Sagami, I.; Shimizu, T. Crucial role of Lys(423) in the electron transfer of neuronal nitric-oxide synthase. J. Biol. Chem., 1999, 274, 26956-26961.

[63] Astashkin, A.V.; Elmore, B.O.; Fan, W.; Guillemette, J.G.; Feng C.J. Pulsed EPR determination of the distance between heme iron and FMN centers in a human inducible nitric oxide synthase. $J$. Am. Chem. Soc., 2010, 132, 12059-12067.

[64] Feng, C. J.; Tollin, G.; Hazzard, J. T.; Nahm, N. J.; Guillemette, J. G.; Salerno, J. C.; Ghosh, D. K. Direct measurement by laser flash photolysis of intraprotein electron transfer in a rat neuronal nitric oxide synthase. J. Am. Chem. Soc., 2007, 129, 5621-5629.

[65] Feng, C. J.; Dupont, A.; Nahm, N.; Spratt, D.; Hazzard, J. T.; Weinberg, J.; Guillemette, J.; Tollin, G.; Ghosh, D. K. Intraprotein electron transfer in inducible nitric oxide synthase holoenzyme. $J$ Biol. Inorg. Chem. 2009, 14, 133-142.

[66] Sempombe, J.; Elmore, B. O.; Sun, X.; Dupont, A.; Ghosh, D. K.; Guillemette, J. G.; Kirk, M. L.; Feng, C. J. Mutations in the FMN domain modulate MCD spectra of the heme site in the oxygenase domain of inducible nitric oxide synthase. J. Am. Chem. Soc., 2009, $131,6940-6941$. 